\title{
Randomized Controlled Trial of Traditional Chinese Medicine (Acupuncture and Tuina) in Cerebral Palsy: Part 1-Any Increase in Seizure in Integrated Acupuncture and Rehabilitation Group Versus Rehabilitation Group?
}

\author{
Yun Wu, M.S., ${ }^{*}$ Li-Ping Zou, M.D.,${ }^{1,2^{*}}$ Tong-Li Han, ${ }^{1}$ Hua Zheng, ${ }^{1}$ \\ Opher Caspi, M.D.,Ph.D., ${ }^{3}$ Virginia Wong, M.D., Ph.D., ${ }^{4}$ Yani Su, ${ }^{5}$ and Kun-Ling Shen, M.D., Ph.D. ${ }^{1}$
}

\begin{abstract}
Objective: The objective of this study was to observe for any change in baseline seizure frequency with acupuncture in children with cerebral palsy.

Methods: A randomized controlled study was conducted: Group I consisted of integrated acupuncture, tuina, and rehabilitation (physiotherapy, occupational therapy, and hydrotherapy) for 12 weeks; and Group II consisted of rehabilitation (physiotherapy, occupational therapy, and hydrotherapy) for 12 weeks. After a washout period of 4 weeks, Group II then received acupuncture and tuina for 12 weeks. Each subject received 5 daily acupuncture sessions per week for 12 weeks (total $=60$ sessions). All children were assessed for any change in seizure frequency during treatment.

Results: One hundred and sixteen (116) children were recruited and randomized into Group I $(N=58)$ and Group II $(N=58)$. Thirty-three (33) children withdrew (9 from Group I and 24 from Group II). Of the remaining 83 children, Group I consisted of 49 and Group II of 34 children. For baseline, 5 children $(6 \% ; 5 / 83)$ had seizures. During phase 1 (12 weeks) of integrative treatment and subsequent 4-week follow-up, 3 children in Group I had seizures. Among those 3 children with seizures, 1 child with prior history of recurrent febrile seizure had 3 more recurrent febrile seizures during acupuncture treatment and 2 children without any prior history of seizures had new-onset seizures (1 with 3 recurrent febrile seizures and 1 with afebrile seizure). For Group I, 2 children with epilepsy had no increase in seizure frequency during acupuncture treatment. For Group II during the phase 2 acupuncture period, none had increase in seizure frequency. In both groups, 4 of 5 children (80\%; 2 in Group I and 2 in Group II) with seizures had no increase in seizure frequency during acupuncture treatment and follow-up.

Conclusions: The risk of increasing seizure is not increased with acupuncture treatment for cerebral palsy.
\end{abstract}

\footnotetext{
${ }^{1}$ Department of Neurology, Beijing Children's Hospital, The Capital Medical University of Medical Sciences, Beijing, China.

${ }^{2}$ Department of Pediatrics, Chinese PLA General Hospital (No. 301 Hospital of PLA), Beijing, China.

${ }^{3}$ The Recanati Center for Medicine and Research, and the Section of Integrative Medicine, Rabin Medical Center (Beilinson Campus), Petah-Tikva, Israel.

${ }^{4}$ Division of Child Neurology/Developmental Paediatrics/NeuroHabilitation, Department of Paediatrics and Adolescent Medicine, University of Hong Kong, Hong Kong, China.

${ }^{5}$ Department of Pediatrics, The University of Arizona, Tucson, AZ.

${ }^{*}$ Co-senior authors.
} 


\section{Introduction}

tudies on the prevalence of epilepsy in cerebral palsy (CP) $\checkmark$ showed that around $28 \%$ of children with $\mathrm{CP}$ have epilepsy. ${ }^{1,2}$ Acupuncture originated in China and has been used for over 2000 years. In 1993, the United States Food and Drug Administration estimated that Americans paid 9 to 12 million visits per year to acupuncture practitioners and spent over 500 million dollars annually on acupuncture treatment.. ${ }^{3}$ Despite its long history, acupuncture is now one of the most common therapeutic techniques that complement modern therapy worldwide. The Chinese reported an exceptionally high response rate with the administration of an integrated package of care that included a combination of intense "conventional" therapies and acupuncture for CP. Despite numerous anecdotal reports, this claim had not yet been studied in a rigorous scientific way.

Acupuncture treatment for children with CP with seizure or epilepsy had been a controversial issue. Lytle suggested that acupuncture can suppress seizures through an inhibitory amino acid in the central nervous system ${ }^{4}$ and electro-acupuncture might inhibit epilepsy via upregulating the concentration of taurine transporter to increase the release of taurine. ${ }^{5}$ Any anticonvulsant effect of acupuncture might be related to the decrease of neuronal and inducible nitric oxide synthases. ${ }^{6}$ Contrary to this, Wu et al. reported that acupuncture might induce seizure or epilepsy in children with CP because the seizure threshold was lower than that in normal children, ${ }^{7}$ and acupuncture has a real and enduring effect on motor cortex functional changes by increasing cortical excitability. ${ }^{8}$

The role of acupuncture therapy in $\mathrm{CP}$ with seizures is still controversial. We therefore collaborated with the Department of Pediatrics at the University of Arizona in October 2004 in a project "Acupuncture as Complementary Therapy for Cerebral Palsy, NCT00221247." This is part 1 of this study and we prospectively evaluated for any change in seizure frequency in $\mathrm{CP}$ undergoing acupuncture treatment.

\section{Materials and Methods}

The study was a parallel, prospective, evaluation-blind, randomized controlled clinical trial (NCT00221247) designed to determine the effectiveness of acupuncture as an adjunct therapy to "conventional" rehabilitation (physical, occupational, and hydrotherapies) (Fig. 1).
In part 1 (this study), we aim to study any change in seizure frequency in children with CP undergoing acupuncture treatment. In part 2, we will analyze for any functional change (gross motor function, fine motor function, range of motion of 6 joints, and function as assessed by the primary caregiver), and this will be reported in another paper.

Between February 2003 and November 2006,116 children had been enrolled in the study.

They were randomized into 2 groups by stratified blocked randomization (Fig. 2). Stratified randomization was done by performing a separate blocked randomization procedure within each of 2 strata: age of the child and CP severity. The baseline data of enrolled children are summarized in Table 1.

\section{Phase 1}

In phase 1 of this study, children assigned to Group I received integrative therapy including electro-acupuncture and rehabilitation (physiotherapy, occupational therapy, and hydrotherapy) for 12 weeks. The children assigned to Group II received only rehabilitation (physiotherapy, occupational therapy, and hydrotherapy) for 12 weeks without acupuncture.

Acupuncture was given on a daily basis, 5 days a week for 12 weeks for a total of 60 treatment sessions for each intervention. Following the 12 weeks of treatment, there was a washout period of 4 weeks when neither group received any treatment.

\section{Phase 2}

Following this washout period, all children in Group II returned for a full 12 weeks' course of acupuncture without rehabilitation (physiotherapy, occupational therapy, and hydrotherapy). Children in Group I will receive no treatment.

\section{TCM approach}

The Traditional Chinese Medicine (TCM) approach to CP consisted of the following: (1) Diagnosis according to syndromal differentiation using the "Eight-Principle Differentiation" model with the TCM practitioner deciding on the pattern or the nosological category for each child and selecting acupoints accordingly; (2) Treatment according to symptoms: paralysis, spasticity, or other CP symptoms. Treatment

\section{4 weeks}

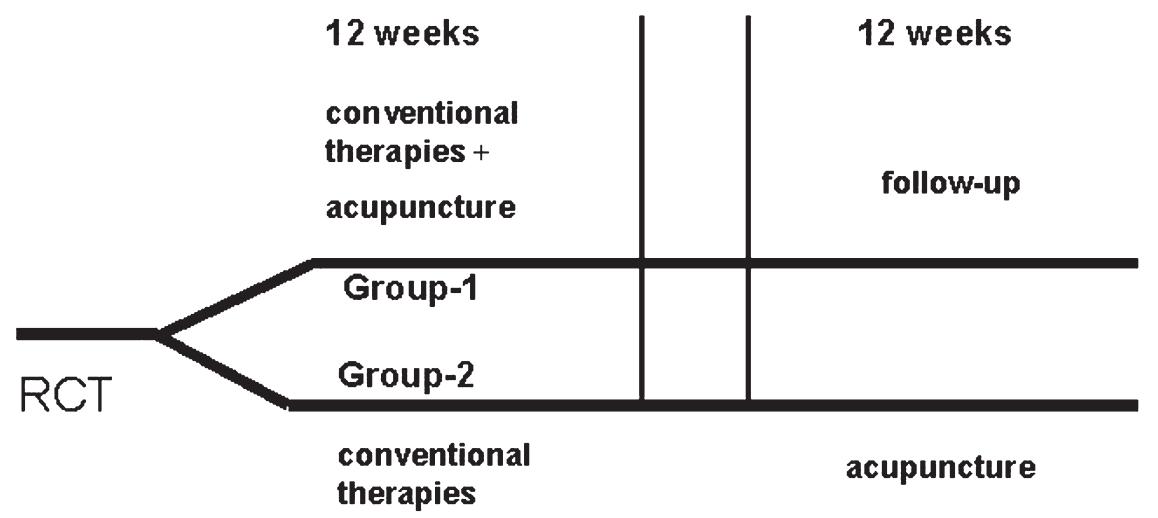

FIG. 1. The overall study design of the clinical trial. RCT, randomized controlled trial. 


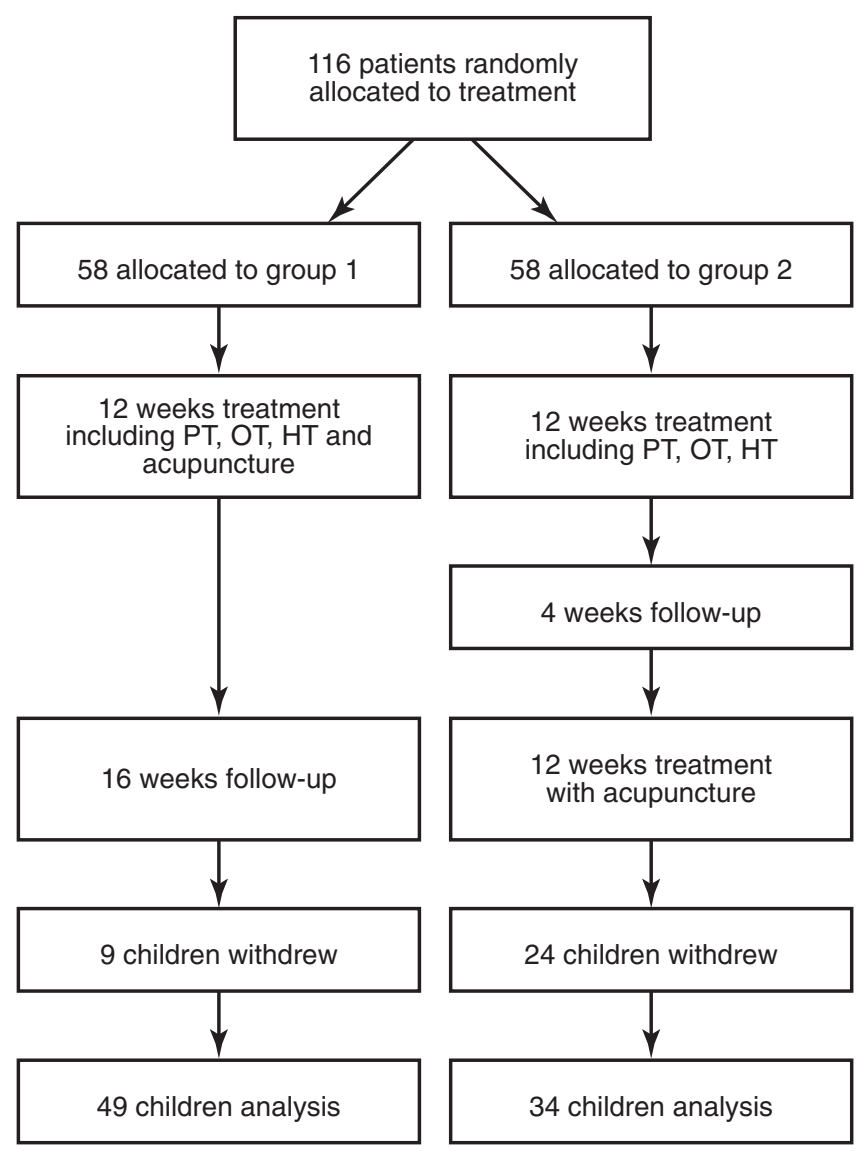

FIG. 2. The flow chart for participants. PT, physiotherapy; OT, occupational therapy; HT, hydrotherapy.

typically consisted of massaging and needling body points with a combination of manual and electrostimulation to treat the paralysis and spasticity by "opening the channels, quickening the blood, and freeing the network vessels"; needling scalp acupuncture areas to increase the strength of remote muscles; and treating other related symptoms as the TCM approach is a holistic medicine. Spastic CP typically was classified in 2 main patterns: dual Deficiency of Liver and Spleen; or Liver and Kidney yin Deficiency.

Once a child with CP is enrolled into the study but before randomization, one of the acupuncturists conducted a baseline evaluation to determine the child's TCM diseases category or pattern on the basis of "Eight-Principle Pattern Differentiation," organ networks and channels involved, taking into consideration the severity, pregnancy, birth, and health history of the mother to determine the etiology; differentiate the TCM nosological category specific to the child, and design a treatment strategy based on TCM principles using an acupuncture protocol. The acupuncturist also observed the patient's demeanor and features, in the context of TCM diagnosis (tongue, face color, eye expression) and observed the Index Finger Vessel in children younger than 3 years or palpated the pulse and channel in those more than 3 years. A detailed record of this evaluation was kept and used for quality assurance and future analyses. On the first session of every week, the practitioner evaluated for any changes and adjusted acupoints and treatment accordingly. Once every 4 weeks during acupuncture treatment, the practitioner would conduct a simplified analysis of this evaluation to systematically evaluate for any change.

Children randomized to Group I received acupuncture immediately upon enrollment (Phase 1), whereas children who had been randomized to Group II received acupuncture 16 weeks later (Phase 2). Each subject received 5 acupuncture sessions during each week for 12 weeks (i.e., total $=60$ sessions). Treatment was designed to address pattern differentiation and symptoms and was individually tailored to each child's differential diagnosis, severity, and stage of disease based on the study acupuncture protocol.

Treatment sessions consisted of a short course of Chinese tuina or massage in affected areas followed by needling of 2-3 acupoints with manual stimulation and needle retention in order to address pattern differentiation; needling of several points on the trunk and limbs to regulate the channels, with manual stimulation and no retention of needles; retention of the needles at 4 points on the affected $\operatorname{limb}(\mathrm{s})$ with electrostimulation; and needling of 2-3 scalp acupoints using manual stimulation and needle retention. When indicated, needles will be retained for 20-30 minutes. Stainless steel disposable needles, $0.30 \mathrm{~mm}$ in diameter and $25 \mathrm{~mm}$ in length, were used in all points.

\section{Body acupuncture}

Body acupuncture was used to address the underlying pattern and regulate circulation of $q i$ and blood in the channels to correct function of the limbs. Needles was inserted and manipulated manually until the phenomenon of "acquisition of energy" or de qi. Either supplementation or drainage techniques was selected based on the targeted therapeutic effect. When addressing the underlying pattern, needles were manually stimulated and retained. If one aimed at regulating the channels, several points were needled manually but the needle was not retained. Once de qi was obtained, the needles were removed. Standard location and nomenclature of acupuncture points was followed. Acupoints were selected on the affected limb(s). After obtaining de qi sensation, electrostimulation was applied to 4 acupoints, which were selected according to channel distribution primarily on the Foot and Arm Yang-Ming (Stomach/Large Intestine) and Shao-Yang (Gall Bladder/Triple Heater) channels. For electrostimulation, static electrical current passed through the acupuncture needles to strengthen the manual stimulation. A moderate intermittent waveform and a pulse frequency of 4-6 Hz was applied for 20-30 minutes, starting with 0 volts, gradually increasing with periodic adjustments according to the tolerance of the child to a maximum of 80 volts. We used the Great Wall brand (model KWD-808) electro-acupuncture stimulator. (Changzhen Wujin Great Wall Medical Device Co., Ltd., Jiang Su, China).

\section{Scalp acupuncture}

Needling was performed on the side opposite to the affected limbs (i.e., on the side of the brain lesion). A total of 2-3 acupuncture scalp acupoints were selected. After the needles were inserted at a $15^{\circ}-30^{\circ}$ angle under the galea aponeurotica, they were retained parallel to the scalp at a secure depth for 30 minutes. Needling techniques in the scalp were selected and modified individually on the basis of etiology and location of the lesion, the symptom differentiation, and the study acupuncture protocol. 
Table 1. Baseline Data of Enrolled Children

\begin{tabular}{|c|c|c|c|c|c|c|c|c|}
\hline & \multicolumn{2}{|c|}{ Characteristics } & \multicolumn{3}{|c|}{ GMFCS level } & \multicolumn{3}{|c|}{ Type of spastic $C P$} \\
\hline & Age in months & Gender & & & & & & \\
\hline & (Mean) & $M / F$ & I & II & III & Hemiplegia & Diplegia & Quadriplegia \\
\hline Group I & 28 & $42 / 16$ & $13 / 58$ & $12 / 58$ & $33 / 58$ & $8 / 58$ & $41 / 58$ & $9 / 58$ \\
\hline Group II & 29 & $34 / 24$ & $20 / 58$ & $20 / 58$ & $18 / 58$ & $10 / 58$ & $40 / 58$ & $8 / 58$ \\
\hline
\end{tabular}

CP, cerebral palsy; GMFCS, Gross Motor Function Classification System; M, male; F, female.

All participating acupuncturists in this study were licensed by the Chinese Ministry of Health. They all had undergraduate acupuncture training for 4 years with more than 20 years of clinical experience.

This international collaborative study was conducted at 2 locations: (1) Beijing Children's Hospital: for case recruitment, intervention therapies, videotaping, and data collection; and (2) the University of Arizona: blind scoring of the videotapes and data analyses; and logistic support was provided to assure the scientific integrity of the study.

Inclusion criteria included children aged 12-72 months with a diagnosis of spastic CP or mixed-type CP where spasticity was the dominant feature.

Exclusion criteria included (1) any damage to the CNS that was not static in nature (e.g., neurodegenerative disease) or occurred after first year, or any chromosomal anomaly; (2) children for whom the diagnosis of spastic CP or mixed-type $\mathrm{CP}$ with spasticity as the dominant feature could not be established with absolute certainty; (2) children with dyskinetic CP; (3) medical conditions, whether acute or chronic, for which acupuncture or intense "conventional" therapies were considered contraindicated; and (4) any children with scheduled treatments during the study period (e.g., orthopedic or neurosurgical procedure, Botulinum toxin injection, a baclofen pump or hyperbaric oxygen treatment).

Children with CP with seizures did not influence the randomization. The frequency of convulsions in 2 groups of children before treatment and during treatment was compared to see whether the acupuncture had any impact on the incidence of seizure activity.

Informed consent was provided by the parents, and the study was approved by the Beijing Children Hospital Subcommittee on Human Studies.

\section{Results}

Of 116 children recruited into our study, 58 each were randomized into Group I and Group II. However, 33 were with- drawn (Group I = 9; Group II = 24). Of 83 enrolled into this study, 49 were analyzed in Group I and 34 in Group II (Fig. 2).

\section{Baseline seizure and electroencephalogram profile (Table 2)}

Group I: There were 3 children with seizures, of which 2 had epilepsy (N1 and N2) and 1 had recurrent febrile seizure (N3).

Group II: there were 2 children with seizures, of which 1 had epilepsy (N4) and 1 had with recurrent febrile seizure (N5).

All 3 children with epilepsy had abnormal electroencephalogram (EEG), including 2 with multifocal epileptic discharges, and 1 with generalized slow waves. Two (2) patients with recurrent febrile seizure had focal epileptic discharges.

For those without clinical seizures $(N=78 / 83), 10$ had subclinical epileptic discharges [Group $1(N=6)$, Group 2 $(N=4)]$.

During treatment (Fig. 1). During phase 1 (12 weeks) of treatment and subsequent 4 weeks follow-up, 3 children had seizures in Group I and none in Group II. Among those 3 children with seizures, 1 child (Table 2: N3) with prior history of recurrent febrile seizure had 3 more recurrent febrile seizures during acupuncture treatment and 2 children without any prior history of seizures had new-onset seizures (1 with 3 recurrent febrile seizures and 1 with afebrile seizure).

None of the children in Group II develop any seizures during phase 2 of acupuncture therapy.

In both groups, 4 children out of 5 (i.e., $80 \%$ ) with seizures had no increase in seizure frequency during acupuncture treatment and follow-up (2 in Group I; 2 in Group II). All 10 children with subclinical epileptic discharges in the EEG did not have seizures during acupuncture treatment.

\section{Discussion}

Our study of acupuncture for CP children did not show any increase in seizure frequency in both clinical and subclinical

Table 2. Characteristics of Seizures Before Treatment

\begin{tabular}{|c|c|c|c|c|c|c|c|}
\hline Number & $\begin{array}{l}\text { Group } \\
\text { number }\end{array}$ & $\begin{array}{l}\text { Gender/ } \\
\text { age }\end{array}$ & $\begin{array}{c}\text { GMFCS } \\
\text { level }\end{array}$ & Type & $\begin{array}{l}\text { Frequency } \\
\quad \text { (total) }\end{array}$ & $\begin{array}{c}\text { Antiepilepsy } \\
\text { drug }\end{array}$ & Diagnosis \\
\hline N1 & Group I & $\mathrm{M} / 26 \mathrm{~m}$ & II & Diplegia & 10 times & VPA & Epilepsy \\
\hline N2 & Group I & $\mathrm{F} / 24 \mathrm{~m}$ & II & Quadriplegia & 2 times & PB & Epilepsy \\
\hline N3 & Group I & $\mathrm{F} / 32 \mathrm{~m}$ & $\mathrm{I}$ & Hemiplegia & 2 times & VPA & Febrile convulsion \\
\hline N4 & Group II & $\mathrm{M} / 60 \mathrm{~m}$ & III & Diplegia & $>20$ times & $\begin{array}{l}\text { VPA treated } \\
\text { before } 24 \mathrm{~m}\end{array}$ & Epilepsy \\
\hline N5 & Group II & $\mathrm{M} / 40 \mathrm{~m}$ & I & Diplegia & 3 times & None & Febrile convulsion \\
\hline
\end{tabular}

M, male; F, female; m, months; GMFCS, Gross Motor Function Classification System; VPA, valproic acid; PB, phenobarbitone. 
settings with a prospective randomized controlled trial extending over 28 weeks $(12+4+12$ weeks). To our knowledge, this is the first prospective, randomized, controlled trial of acupuncture for children with $\mathrm{CP}$ in assessing the risk of increasing seizure frequency. This paper only evaluates any change in seizure frequency during and after acupuncture, and a larger study will be reported in another paper.

It is to be noted that acupuncture is safe in treating children with $\mathrm{CP}$ with seizure, and even for those with subclinical epileptiform discharges in the EEG. However, as our cohort of CP children had been on anticonvulsants and the seizures had been relatively well under control with monotherapy, one still has to be cautious when conducting acupuncture for any $\mathrm{CP}$ with intractable epilepsy. Our study had been preliminary, and $\mathrm{CP}$ children with poorly controlled seizures (e.g., daily seizures, polytherapy) should be included in the future to assess whether acupuncture is indeed safe. Although the frequency of seizure decreased after acupuncture in only 2 cases while on acupuncture, this may just be due to the natural course of the disease. However, we did not know whether acupuncture might have any role in the treatment of seizures.

Acupuncture is a traditional method of treatment rooted in our ancient Chinese culture that has existed for at least 2 millennia.9,10 According to Chinese philosophy, acupuncture techniques are based on the hypothesis of meridians and energy flow. TCM has proposed various therapeutic theories and practical experience for epilepsy, of which acupuncture and herbs had been investigated. ${ }^{11,12}$

The mechanism of acupuncture for treating epilepsy is not clearly understood. Some hypothesized that electro-acupuncture improved epileptic seizures induced by kainic acid in taurine-depletion rats. ${ }^{4}$ However, Nakken has not shown any beneficial effect of acupuncture in chronic intractable epilepsy as the decrease in seizure frequency did not reach statistical significance and there was an increase in the number of seizurefree weeks in both acupuncture and sham acupuncture groups, and reached statistical significance in the sham group. ${ }^{10}$

The limitation of our study is that this is not an intentionto-treat analysis as we did not analyze the 33 cases that withdrew. However, based on the remaining 83 cases with baseline EEG performed, there was no aggravation of seizures even in the 10 cases with subclinical epileptic discharges. However, there were 2 children $(2.4 \% ; 2 / 83)$ without any prior history of seizures and having normal EEG but who had new-onset seizures (1 with 3 recurrent febrile seizures and 1 with afebrile seizure). As recurrent febrile seizure is common for young children, one can only be cautious and vigilant for the development of febrile or afebrile seizure for any child with CP undergoing any therapy.

Thus, we conclude that acupuncture is a safe treatment option as integrated therapy for children with CP with wellcontrolled epilepsy or recurrent febrile seizures. We did not find any evidence of acupuncture therapy in increasing seizure in our cohort. However, one still has to be vigilant for the development of any febrile or afebrile seizure for any child with CP undergoing any therapy including acupuncture. Acupuncture can be safe in treating $\mathrm{CP}$ with underlying epilepsy that is well controlled with anticonvulsants.

\section{Acknowledgments}

This study was supported by Arizona government research funds (Y410503), the International Scientific and Tech- nological Cooperation Foundation of Beijing (grant no. 2007G05), and the Beijing Chinese Medicine projects (grant no. JJ2005-17). We are indebted to all patients who participated in this study and all clinicians involved in the collection of blood samples.

\section{Disclosure Statement}

No competing financial interests exist.

\section{References}

1. Hadjipanayis A, Hadjichristodoulou C, Youroukos S. Epilepsy in patients with cerebral palsy. Dev Med Child Neurol 1997;39:659-663.

2. Andersen GL, Irgens LM, Haagaas I, et al. Cerebral palsy in Norway: Prevalence, subtypes and severity. Eur J Paediatr Neurol 2008;12:4-13.

3. Lytle CD. An Overview of Acupuncture. Washington, DC: United States Department of Health and Human Services, Health Sciences Branch, Division of Life Sciences, Office of Science and Technology, Center for Devices and Radiological Health, Food and Drug Administration, 1993.

4. Jin $\mathrm{HB}, \mathrm{Li} \mathrm{B}, \mathrm{Gu}$ J, et al. Electro-acupuncture improves epileptic seizures induced by kainic acid in taurine-depletion rats. Acupunct Electrother Res 2005;30:207-217.

5. Li Q, Guo JC, Jin HB, et al. Involvement of taurine in penicillin-induced epilepsy and anti-convulsion of acupuncture: A preliminary report. Acupunct Electrother Res 2005; 30:1-14.

6. Yang R, Huang ZN, Cheng JS. Anticonvulsion effect of acupuncture might be related to the decrease of neuronal and inducible nitric oxide synthases. Acupunct Electrother Res 1999;24:161-167.

7. Wu 1, Gao C£ $£^{\mathrm{TM} M a} \mathrm{C}-Y$. Principles of rehabilitation treatment for the cerebral palsy children complicated with epilepsy [in Chinese]. Chin J Rehabil Theory Pract 2006;12: 97-98.

8. Lo YL, Cui SL, Fook-Chong S. The effect of acupuncture on motor cortex excitability and plasticity. Neurosci Lett 2005;384:145-149.

9. Jayasuriya A. Clinical Acupuncture, A to Z Acupuncture Course. 7th ed. Shahdara, Delhi: B Jain Publishers (P), 1998.

10. Lai CW, Lai YH. History of epilepsy in Chinese traditional medicine. Epilepsia 1991;32:299-302.

11. Wu D. Suppression of epileptic seizures with acupuncture: Efficacy, mechanism and perspective. Am J Acupunct 1988; 16:113-117.

12. Cheuk DK, Wong V. Acupuncture for epilepsy. Cochrane Database Syst Rev 2006;2:CD005062.

Address reprint requests to:

Li-Ping Zou, M.D.

Department of Pediatrics

Chinese PLA General Hospital

No. 301 Hospital of PLA

and Department of Neurology

Beijing Children's Hospital

The Capital University of Medical Sciences

Beijing 100045

China

E-mail: zouliping21@hotmail.com 